\title{
O conhecimento de idosos portadores de doenças crônicas não transmissíveis acerca de seu tratamento polimedicamentoso
}

\section{The knowledge of elderly carriers of noncommunicable diseases about their polymedicate treatment}

\section{El conocimiento de idosos portadores de enfermedades crónicas no transmisibles acerca de su tratamiento polimedicamentoso}

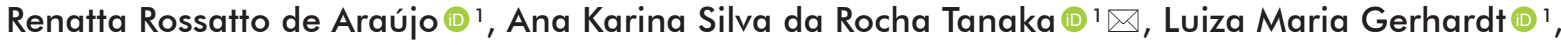 \\ Maria Luiza Paz Machado (1) 1
}

Universidade Federal do Rio Grande do Sul (UFRGS). Porto Alegre, RS, Brasil.

\section{ARTICLE INFO}

\section{Article history}

Received: 29/09/2019

Accepted: $28 / 05 / 2019$

Published: 29/08/2019

\section{$\triangle$ Correspondent Author}

Ana Karina Silva da Rocha Tanaka

Escola de Enfermagem - UFRGS

Rua São Manoel 963, Santana

90.620-001, Porto Alegre, RS, Brasil

ana.tanaka@ufrgs.br

\section{C) 2019 All rights reserved}

\section{Editors}

Alfredo Cataldo Neto

Newton Luiz Terra

Assistant Editors

Paula Engroff

Vanessa Sgnaolin

\begin{abstract}
RESUMO
OBJETIVO: Avaliar o conhecimento dos pacientes idosos com doenças crônicas não transmissíveis acerca de seu tratamento polimedicamentoso.

METODOLOGIA: Estudo exploratório descritivo com abordagem qualitativa. A amostra do estudo foi intencional, composta por 14 pacientes idosos. A coleta dos dados foi realizada no período compreendido entre setembro e dezembro de 2017. A pesquisa foi realizada em um hospital de grande porte no Rio Grande do Sul, os pacientes estavamvinculados à agenda de Enfermagem Adulto Diabético (EAD) ambulatorial, através das consultas de enfermagem. Os dados foram analisados conforme a temática de Minayo.

RESULTADOS: A análise resultou em duas categorias denominadas: $\bigcirc$ conhecimento acerca da doença e do tratamento farmacológico e $\bigcirc$ uso das plantas medicinais. Os resultados evidenciaram que: todoseram portadores de três ou mais doenças crônicas não transmissíveis com prevalência para Diabetes Mellitus tipo 2 e Hipertensão Arterial Sistêmica; mais de cinquenta por cento (50\%) utilizava oito ou mais medicamentos concomitantes; todos faziam uso de plantas medicinais concomitantes ao tratamento medicamentoso.

CONCLUSÕES: Conclui-se que os pacientes demonstraram um entendimento leigosobre suas doenças e um baixo entendimento do tratamento medicamentoso, o que pode ter como consequência o risco de não adesão ao tratamento e o aumento das possibilidades de ocorrência de reações adversas aos medicamentos.
\end{abstract}

DESCRITORES: Enfermagem; Doenças crônicas não transmissíveis; Idoso; Polimedicação; Segurança do paciente; Cooperação do paciente.

\section{ABSTRACT}

OBJECTIVE: To evaluate the knowledge of elderly patients with chronic noncommunicable diseases about their treatment.

METHODOLOGY: A descriptive exploratory study with a qualitative approach. The study sample was intentional, composed of 14 elderly patients, of both sexes, linked to the Diabetic Adult Nursing (EAD) agenda of the ambulatory of the Clinical Hospital of Porto Alegre. Data collection was carried out between September and December 2017.

Results: The analysis resulted in two categories: Knowledge about the disease and pharmacological treatment and Use of medicinal plants. The results showed that: all had three or more noncommunicable diseases, with prevalence for Diabetes Mellitus and Systemic Arterial Hypertension; more than half used eight or more concomitant medications; all used medicinal plants concomitant with drug treatment.

CONCLUSIONS: Patients have shown a lay understanding of their illnesses and a poor understanding of drug treatment, which may result in the risk of non-adherence to treatment and increased chances of adverse drug reactions.

KeYwordS: Nursing; Noncommunicable diseases; Elderly; Polyimedication; Patient safety; Patient compliance. 


\section{RESUMEN}

OBJETIVO: Evaluar el conocimiento de los pacientes ancianos con enfermedades crónicas no transmisibles acerca de su tratamiento polimedicamento. METODOLOGÍA: Estudio exploratorio descriptivo con abordaje cualitativo. La encuesta fue intencional, compuesta por 14 pacientes ancianos. La recolección de los datos fue realizada en el período comprendido entre septiembre y diciembre de 2017 . La investigación fue realizada en un hospital de gran porte en Rio Grande do Sul, los pacientes estaban vinculados a la agenda de Enfermería Adulto Diabético (EAD) ambulatorial, a través de las consultas de enfermería. Los datos fueron analizados conforme a la temática de Minayo.

RESULTADOS: El análisis resultó en dos categorías denominadas: El conocimiento acerca de la enfermedad y del tratamiento farmacológico y El uso de las plantas medicinales. Los resultados evidenciaron que: todos los portadores de tres o más enfermedades crónicas no transmisibles con prevalencia para Diabetes Mellitus tipo 2 y Hipertensión Arterial Sistémica; más del cincuenta por ciento (50\%) utilizaba ocho o más medicamentos concomitantes; todos hacían uso de plantas medicinales concomitantes al tratamiento medicamentoso.

CONCLUSIONES: Se concluye que los pacientes demostraron un entendimiento laico de sus enfermedades y un bajo entendimiento del tratamiento medicamentoso, lo que puede tener como consecuencia el riesgo de no adhesión al tratamiento y el aumento de las posibilidades de ocurrencia de reacciones adversas a los medicamentos.

PALABRAS CLAVE: Enfermería; Enfermedades crónicas no transmisibles; Personas de edad avanzada; Polifarmacia; Seguridad del paciente; Cooperación del paciente.

\section{INTRODUÇÃO}

O processo de envelhecimento é marcado por diversas alterações fisiológicas relacionadas com o aumento da idade, sendo necessário adotar medidas que garantam o acesso e o uso racional dos medicamentos. Para isso, devem ser realizadas revisões da terapia, com garantia de adesão ao tratamento; limitações da prescrição de fármacos que podem causar danos, avaliações do custo/ benefício do tratamento e identificação de medicamentos inapropriados e/ou desnecessários. ${ }^{1}$

Com o envelhecimento, o desenvolvimento de doenças crônicas não transmissíveis (DCNT) vêm se tornando cada vez mais presente na população, tornando-se um grande desafio de saúde pública, principalmente pela morbidade e mortalidade que causam. Estas doenças podem ser prevenidas e causam sérios graus de limitações que afetam tanto o cotidiano e o bem-estar do indivíduo quanto à economia do país. São doenças que acometem principalmente idosos e que, observando-se o crescimento do número da população idosa brasileira, nota-se a necessidade de investigação e análise das ações de prevenção e promoção à saúde. ${ }^{2}$

A morte prematura consequentes das DCNT continuam sendo um dos principais problemas no último século. Estas comorbidades afetam principalmente as populações mais pobres e desfavorecidas. $\mathrm{O}$ aumento da prevalência das DCNT tem sua origem ligada a pobreza, globalização da promoção e no comércio de produtos nocivos à saúde, na célere urbanização e no crescimento populacional. ${ }^{3}$

Diante disto, o Brasil vem trazendo políticas e/ou estratégias mais direcionadas como a rede de atenção às pessoas com DCNT. Comprovações científicas para a adesão ao tratamento medicamentoso por pacientes portadores de DCNT podem subsidiar as decisões na política de saúde em nível nacional e serem úteis na implementação das ações direcionadas no nível local. ${ }^{4}$

$\mathrm{O}$ aumento do acesso aos medicamentos, como parte do cuidado integral aos portadores de DCNT, está inserido nas estratégias implementadas, levando em conta que o tratamento medicamentoso proporciona o controle das doenças, diminuição da morbimortalidade e melhora da qualidade de vida dos usuários portadores de diversos estados de saúde. ${ }^{5}$

Pacientes idosos mal orientados quanto à frequência e/ou a duração do tratamento medicamentoso, podem acabar desenvolvendo outras comorbidades além das que já possui.Inúmeros são os fatores que podem interferir na adesão ao tratamento, dentre eles os culturais, a origem da população estudada, a relação com a equipe de saúde e com a família e que em cada 10 portadores de doenças crônicas, cerca de 3 apresentam alguma característica de descontinuidade da terapia medicamentosa, podendo decorrer de orientação inadequada. ${ }^{6}$

A inefetividade do tratamento medicamentoso pode ser explicada, entre outros fatores, por barreiras de acesso aos serviços de saúde e aos medicamentos, pela ineficácia dos fármacos e, sobretudo, pela não adesão à terapia. ${ }^{7}$ Além da mortalidade, as DCNT demonstram diversas morbidades relacionadas, refletindo em um grande número de internações, assim como estão entre as principais causas de amputações e de perdas de mobilidade e de outras funções neurológicas. Consequentemente, também acabam afetando a perda significativa da qualidade de vida, que piora à medida que a doença se agrava. ${ }^{8}$ 
A polimedicação/polifarmácia, o uso simultâneo e de forma crônica de fármacos para doenças e sintomas distintos pelo mesmo indivíduo, prática cada vez mais frequente em idosos, aumenta a probabilidade de reações adversas ao medicamento, interações medicamentosas e diminuição da adesão ao tratamento. ${ }^{9}$ Dessa forma, percebe-se que há deficiência quanto à orientação aos pacientes. Essas lacunas no conhecimento do tratamento afetam diretamente a adesão - elas poderiam ser suprimidas por uma abordagem compartilhada da doença entre profissional da saúde e paciente. ${ }^{10}$

Diversos são os fatores que contribuem para a não adesão ao tratamento, tais como, situação financeiras, muitos medicamentos prescritos, plano terapêutico prescrito, efeitos adversos e reações adversas aos medicamentos, a dificuldade de acesso ao sistema de saúde, a característica assintomática da doença e a sua cronicidade. ${ }^{11}$ Outros fatores como consumo elevado e uso prolongado, desconhecimento sobre os medicamentos e de sua condição de saúde, falta de motivação, baixa escolaridade, falta de acompanhamento pela família/cuidador, falta de monitoramento pela equipe de saúde e idade avançada, também podem estar associados a não adesão ao tratamento. ${ }^{12}$

Nesse contexto, o objetivo deste trabalho foi: avaliar o conhecimento dos pacientes idosos com DCNT acerca de seu tratamento polimedicamentoso.

\section{MÉTODOS}

Trata-se de um estudo exploratório descritivo com abordagem qualitativa. ${ }^{13,14} \mathrm{O}$ estudo foi realizado no ambulatório de um hospital de grande porte no Rio grande do Sul, através de consultas de enfermagem realizadas por agenda de Enfermagem Adulto Diabético (EAD). Esta agenda é caracterizada por acompanhar pacientes adultos e idosos portadores de diabetes mellitus tipo 2. A amostra do estudo foi intencional, composta por pacientes idosos, de ambos os sexos, vinculados à agenda de EAD. O dimensionamento da quantidade de entrevistas foi o critério de saturação, considerando um número suficiente de participantes para permitir reincidência e complementaridade das informações. ${ }^{13}$ Com isso, encerrou-se a coleta após 14 pacientes entrevistados.

Foram considerados critérios de inclusão: ser idoso, portador de pelo menos uma DCNT; utilizar no mínimo cinco medicamentos simultaneamente ${ }^{15,16}$; ter condições de manter um diálogo e estar em acompanhamento na agenda EAD.

Os participantes foram convidados a participar de uma entrevista semiestruturada. $\mathrm{O}$ convite ocorreu no dia das consultas de enfermagem de rotina. Aos que aceitaram fazer parte da pesquisa, foi proposto que a entrevista fosse realizada no mesmo dia da consulta. Buscando proporcionar um ambiente calmo, confortável e privativo, as entrevistas foram realizadas em uma sala do ambulatório, previamente agendada. Esta estratégia se justifica pelo fato da maioria dos idosos atendidos na agenda possuírem dificuldade de locomoção, assim como residirem distante do hospital.

Para a interpretação dos resultados e análise de conteúdo, foi utilizado à análise temática por ser a mais adequada à investigação de fenômenos sobre saúde. Esta consiste em descobrir os núcleos de sentido e conduz à contagem de frequência das unidades de significação, as quais definem o caráter do discurso. ${ }^{13}$

$\mathrm{O}$ projeto foi elaborado em consonância com a Resolução nº466/2012 do Conselho Nacional de Saúde (CNS), no que tange a pesquisa com seres humanos. Esta pesquisa foi aprovada via plataforma Brasil, comitê de ética da instituição que foi realizada a pesquisa e pelo comitê de ética da universidade. Número do CEP: 2.270.844 de CAEE:74150817.4.0000.5327.

Os dados coletados durante a pesquisa foram sempre tratados confidencialmente. Os resultados foram apresentados de forma conjunta, sem a identificação dos participantes. Os participantes e os pesquisadores assinaram duas vias iguais do Termo de Consentimento Livre e Esclarecido (TCLE), o qual assegura a participação voluntária, a garantia de anonimato e a possibilidade de desistência a qualquer momento, sem prejuízos dos mesmos. Em caso de os idosos estarem com acompanhantes, esses também foram orientados quanto aos objetivos do estudo.

\section{RESULTADOS}

Esta seção tem como finalidade caracterizar os sujeitos estudados, bem como apresentar as categorias analíticas oriundas da interpretação dos dados, obtidos a partir das entrevistas efetuadas como os mesmos.

Quanto aos 14 pacientes entrevistados, identificou-se que: idade variou entre 60 e 82 anos (média de 69,5 anos, desvio padrão de 6,8); seis eram homens e oito mulheres; três tinham o ensino fundamental incompleto, dois ensino fundamental completo, três ensino médio incompleto, cinco ensino médio completo e um com ensino superior incompleto; sete eram casados, dois separados e cinco viúvos; quatro moravam sozinhos, dois com o cônjuge, três com os filhos e cinco com cônjuge e filhos; todos portavam três ou mais DCNT, com prevalência para Diabetes Mellitus Tipo 2 (DM2) e Hipertensão Arterial 
Sistêmica (HAS); dois pacientes faziam uso de cinco medicamentos concomitantes, dois pacientes faziam uso de seis medicamentos, dois pacientes faziam uso de sete medicamentos e oito pacientes faziam uso de oito medicamentos ou mais; os medicamentos mais utilizados pelos pacientes foram: Sinvastatina (53,3\%); Metformina (46,7\%); Ácido Acetilsalicílico (AAS) e Hidroclorotiazida (HCTZ) (33,3\%); Losartana (26,7\%); Glibenclamida, Captopril, Paracetamol, Levotiroxina e Amitriptilina (20,0\%) (Tabela 1).

Os pacientes foram questionados sobre o conhecimento referente às suas doenças. Diante disto, pode-se constatar que, embora este esteja fundamentado em um conhecimento leigo, os pacientes demonstram algum entendimento sobre as doenças de que são portadores, bem como as possíveis complicações que podem advir das mesmas:

"Eu fumei também, por um bom tempo, mas parei em 2003. Tive um AVC em 93 e eu não tive infarto, era reação do enalapril. Assim que parei de tomar, não deu mais." (P1)

"Diabetes, pressão alta, e agora problema nos rins [...] não funcionam mais tão bem. Provavelmente pelo diabetes." (P8)

Após identificar o conhecimento diante de suas comorbidades, foi questionado quais medicamentos utilizavam para cada uma delas. Por conseguinte, observou-se que alguns participantes possuem um escasso conhecimento frente à função de cada medicamento, como tomar e quais as reações adversas que podem ocorrer.

Os depoimentos a seguir evidenciam o pouco conhecimento sobre a função de cada medicamento:

"Eu não! Ela diz (a médica) esse é pro coração, esse pra diabete, pra tireoide... O omeprazol é pro estômago né? [...] eu tomo muito remédio." (P2)

"Não sei. Só tomo o que me prescrevem e tá ótimo." (P5)

Contudo, alguns deles demonstraram conhecer os medicamentos que utilizam e saber a função de cada um:

"Omeprazol pro estômago. Glibenclamida, metformina e a insulina pra diabetes. A atensina pra pressão. $O$ cilostazol e o AAS pra circulação, afinar o sangue né? Sinvastatina é pra circulação e pro colesterol. Paracetamol e a Dipirona pra dor. Amitriptilina é pro humor" (P1)

"A maioria é pra pressão né, e a Insulina pra diabetes... ah, e a prednisona pra alergia." (P10)

Tabela 1. Prevalência de medicamentos usados pelos pacientes.

\begin{tabular}{|c|c|c|}
\hline Medicamento & $\mathbf{N}$ & $\%$ \\
\hline \multicolumn{3}{|c|}{ Anti-hiperglicemiantes } \\
\hline Metformina & 7 & 46,7 \\
\hline Insulina NPH & 4 & 26,7 \\
\hline Glibenclamida & 3 & 20,0 \\
\hline Glifage & 3 & 20,0 \\
\hline Insulina regular & 2 & 13,3 \\
\hline Acarbose & 1 & 6,7 \\
\hline Insulina Glargina & 1 & 6,7 \\
\hline Insulina Lispro & 1 & 6,7 \\
\hline \multicolumn{3}{|l|}{ Anti-hipertensivos } \\
\hline HCTZ & 5 & 33,3 \\
\hline Losartana & 4 & 26,7 \\
\hline Captopril & 3 & 20,0 \\
\hline Hidralazina & 2 & 13,3 \\
\hline Propanolol & 2 & 13,3 \\
\hline Atensina & 1 & 6,7 \\
\hline Clortalidona & 1 & 6,7 \\
\hline Carvedilol & 1 & 6,7 \\
\hline Enalapril & 1 & 6,7 \\
\hline Metildopa & 1 & 6,7 \\
\hline Propanolol & 1 & 6,7 \\
\hline \multicolumn{3}{|l|}{ Hipolipemiantes } \\
\hline Sinvastatina & 8 & 53,3 \\
\hline Fenofibrato & 1 & 6,7 \\
\hline \multicolumn{3}{|l|}{ Vasculares } \\
\hline AAS & 5 & 33,3 \\
\hline Anlodipina & 2 & 13,3 \\
\hline Cilostazol & 1 & 6,7 \\
\hline Propatinil & 1 & 6,7 \\
\hline Isossorbida & 1 & 6,7 \\
\hline Varfarina & 1 & 6,7 \\
\hline Xarelto & 1 & 6,7 \\
\hline Propafenona & 1 & 6,7 \\
\hline \multicolumn{3}{|l|}{ Dor } \\
\hline Paracetamol & 3 & 20,0 \\
\hline Dipirona & 2 & 13,3 \\
\hline Dorflex & 2 & 13,3 \\
\hline Codeína & 1 & 6,7 \\
\hline \multicolumn{3}{|l|}{ Hipotireoidismo } \\
\hline Levotiroxina & 3 & 20,0 \\
\hline Metimazol & 1 & 6,7 \\
\hline Tapazol & 1 & 6,7 \\
\hline \multicolumn{3}{|l|}{ Depressão } \\
\hline Amitriptilina & 3 & 20,0 \\
\hline Furosemida & 2 & 13,3 \\
\hline \multicolumn{3}{|l|}{ Outros } \\
\hline Omeprazol & 3 & 20,0 \\
\hline Acitretina & 1 & 6,7 \\
\hline Alopurinol & 1 & 6,7 \\
\hline Azatioprina & 1 & 6,7 \\
\hline Prednisona & 1 & 6,7 \\
\hline Sabultamol & 1 & 6,7 \\
\hline
\end{tabular}


Ao abordar a forma de tomar os medicamentos, pode-se constatar que a maioria dos pacientes prefere tomar somente com água, em razão de temer interação entre o líquido utilizado e as medicações:

"Não gosto de tomar com café, algo quente, suco... tem que ser água." (P2)

"Sempre só com água. dizem que não é bom tomar com outras coisas juntos, que interfere." (P3)

Os depoimentos dos participantes da pesquisa evidenciaram a pouca informação sobre a maneira correta de ingerir os medicamentos. Este fato pode estar relacionado à complexidade da polimedicação, à falta de orientação por parte do prescritor ou a dificuldade de entendimento por parte do paciente.

Frente ao indício do escasso conhecimento quanto à forma de utilização dos medicamentos prescritos, os participantes foram questionados quanto à ocorrência de sintomas após a ingestão dos mesmos.

"Eu usava o captopril e me dava muita tosse, mas a médica trocou pelo propranolol." (P2)

"A Metformina e Glibenclamida, quando tomadas juntas, me dava muita diarreia e dor de estômago." (P8)

Além do conhecimento acerca das comorbidades e aspectos relacionados ao tratamento medicamentoso, os participantes da pesquisa foram questionados sobre as estratégias utilizadas para o cumprimento da prescrição, as quais possuem a finalidade de evitar o esquecimento da ingestão na hora e dose recomendada.

Os relatos evidenciaram que todos os pacientes entrevistados costumam utilizar algum tipo de organizador, no qual mantêm todos os medicamentos juntos. Entre as estratégias destaca-se: manter as embalagens em cima da mesa; guardar medicamentos nas embalagens em uma caixa de sapato com a prescrição dentro; organizar todos os medicamentos do dia, ou para o dia seguinte, em um organizador conforme turno; nos casos de meio comprimido, cortar todos e guardar num pote.

"Pela caixa. A receita fica sempre junto com as medicações numa caixa de sapato." (P9).

"Eu tenho uma tesoura que corto as medicações que são metade. Dai corto todas logo que pego a cartela e ponho num potinho. Assim já fica pronto pro mês todo." (P13).

Mesmo sendo identificadas estratégias que auxiliam no seguimento adequado da prescrição, evidenciaram-se também algumas condutas não seguras, uma vez que alguns pacientes relataram que por usarem os medicamentos há tanto tempo, já identificam os mesmos pela cor e formato. Esta estratégia é arriscada devido ao grande risco de erro na administração, visto que muitos medicamentos possuem forma e cores semelhantes.

"Eu tenho uma cestinha que coloco todos lá dentro, dentro das suas caixas. Mas eu tomo há anos, tô tão acostumada que já vou direto já sei de cor qual é cada um até pelo tamanho e cor. Quando eu tava tomando codeina, ele era meio parecido com o paracetamol né, daí aquele dali eu cuidava." (P1)

"Pelo formato. Há anos mando manipular todos no mesmo laboratório. Então além das embalagens, se estiverem soltos em cima da mesa, por exemplo, sei como é cada um." (P6)

Por fazerem o tratamento medicamentoso há muitos anos, alguns pacientes relataram que quase nunca deixam de tomar o medicamento, o que pode demonstrar uma boa adesão ao tratamento medicamentoso dessa população.

\section{"Mas eu nunca me esqueço (risos). Sério. Posso me esquecer de comer, mas dos remédios não." (P1) \\ "Muito difícil. Nunca me esqueci. Só as vezes que eu saio e não levo. Geralmente os do meio dia, mas dai quando chego eu tomo. Às vezes o da noite eu quase esqueço, porque não gosto de tomar com a comida, mas minha filha sempre lembra." (P2)}

Também foram relatadas situações em que os medicamentos foram deixados de tomar no horário prescrito. Nesta situação, costumam tomar atrasado (atrasando os demais horários) ou simplesmente suprimem aquela dose, evidenciando novamente uma conduta pouco segura e que pode ter consequências para a efetividade do tratamento:

"Não esqueço. Muito difícil, ainda mais por que minha esposa toma também, dai um lembra o outro. O que às vezes acontece é de tomar atrasado." (P9)

"Raramente me esqueço. Geralmente levo junto. Mas quando acontece, tomo atrasado ou não tomo naquele turno." (P3)

“Tomo atrasado. Vou atrasando tudo dai." (P12)

$\mathrm{Na}$ análise das entrevistas foi possível identificar a utilização de plantas medicinais concomitantes ao tratamento medicamentoso. Frente ao uso de substâncias não prescritas, pode-se constatar um grande uso de chás de ervas caseiro para tratamento de alguma patologia ou apenas para o bem estar. 
"E tem os que tenho plantado em casa: poejo, hortelã, alecrim, um que é pra diabetes, tem uma folha fininha, acho que é insulina o nome. Também tenho cidró, sálvia. Gosto que fazer com casca de maçã e de laranja também...Gosto de por na comida - alecrim, sálvia, orégano, açafrão. O chimarrão eu tomo com mais dois ou três, mas $1 x$ por dia." (P1)

"Às vezes chimarrão, mas duas cuias e já tá bom, se tomo mais dá muita "mijadeira". Gosto de chá de fruta, de caixinha. Mas eu tenho em casa funcho, gervão [...] às vezes tomo de funcho, mas eu gosto mesmo de anis." (P2)

"Chimarrão com ervas (casca de frutas, hortelã, anis, camomila, espinheira santa) e quando estou mal do estômago tomo chá de macela." (P9)

O uso consciente de plantas medicinais, em conjunto com o cultivo sustentável e reconhecimento das espécies para correta aplicação e manipulação, se torna essencial para o sucesso do tratamento e, neste contexto, profissionais de saúde precisam estar engajados, principalmente aqueles ligados aos serviços de atenção primária à saúde.

\section{DISCUSSÃO}

A efetividade do tratamento para DCNT, com controle apropriado de complicações a curto e longo prazo, não depende apenas da intervenção médica. ${ }^{17}$ Os conhecimentos, assim como as crenças do paciente sobre sua condição, influenciam o processo de educação dos pacientes e seus parentes e constitui ponto indispensável no tratamento. Deste modo se nota que a comunicação é um dos principais meios do enfermeiro lidar com a espiritualidade do paciente, estabelecendo um vínculo de confiança, onde possa se sentir à vontade para se expressar suas crenças, sabendo que será compreendido e apoiado. Antes de se planejar alguma ação visando a melhoria do atendimento aos pacientes, é importante conhecer seu nível de entendimento sobre o próprio estado de saúde. ${ }^{17,10}$

O apropriado nível de conhecimento dos pacientes sobre o tratamento é considerado um fator essencial para a utilização dos medicamentos conforme a prescrição médica. ${ }^{16}$ É importante que os mesmos tenham conhecimento e compreendam claramente a enfermidade que os acomete, os objetivos do tratamento proposto, a percepção dos benefícios e possíveis reações adversas do tratamento medicamentoso. ${ }^{18}$ Diante dos resultados, notou-se que a maioria dos pacientes tinha um escasso conhecimento de seus medicamentos, o que pode estar associado a um maior risco de não adesão ou tomar de forma incorreta.
Os idosos são mais suscetíveis aos problemas decorrentesdo uso de diversos medicamentos, principalmente devido a complexidade dos problemas clínicos, tornando-se necessário uma numerosa quantidade de agentes terapêuticos e às alterações farmacocinéticas e farmacodinâmicas características do envelhecimento. ${ }^{19}$ Esta prática ligada ao sistema fisiológico com função reduzida, alterações na excreção, no metabolismo, identificação incorreta do medicamento, via incorreta de administração e armazenamento inadequado que podem ocorrer com o idoso, acabam influenciando a uma desregulação fisiológica do organismo e possível intoxicação. ${ }^{20}$

O idoso é um paciente especial, sendo necessário, em muitos casos, ajustar a terapêutica frente às alterações físicas, fisiológicas ou até psicológicas que este apresenta. $O$ pressuposto é garantir que o idoso obtenha uma adesão total à terapêutica prevenindo ainda que sofra reações adversas aos medicamentos. ${ }^{1,10}$

Após a ingestão de alimentos ou líquidos o pH do estômago se eleva, afetando a desintegração das cápsulas, drágeas ou comprimidos e consequentemente a absorção do princípio ativo. Neste sentido, o consumo simultâneo dos fármacos com bebidas quentes pode causar a destruição prematura desses invólucros expondo as drogas a um $\mathrm{pH}$ a que são sensíveis. ${ }^{21}$ Segundo Torriani ${ }^{22}$, alguns medicamentos como: AAS, captopril, paracetamol, levotiroxina, hidroclorotiazida, tem sua absorção diminuída quando ingeridas antes das refeições. Entretanto, medicamentos como a metformina, alimentos favorecem sua absorção. Com o envelhecimento, percebe-se uma discreta elevação do tempo de esvaziamento gástrico (principalmente para líquidos), todavia este fenômeno ainda é questionável, podendo levar a um prejuízoda absorção de medicamentos dependentes da exposição prolongada ao meio ácido. ${ }^{23}$

Com base nas reações verbalizadas, constatou-se que todas eram reações adversas esperadas conforme Torriani ${ }^{22}$, como diarreia, tosse e dores abdominais. Embora se saiba que alguns sintomas são comuns a alguns medicamentos, é fundamental que os pacientes sejam informados desta possibilidade e de como agir nesta situação. Caso contrário, os sintomas relatados podem se tornar um dos motivos para a má adesão ao tratamento farmacológico.

A adesão ao tratamento é influenciada pela forma em que os medicamentos são usados, levando em conta os horários, via de administração, medicamento e dose correta, assim como a quantidade de medicamentos utilizados. Estes fatores podem levar a um aumento da sensação de ausência de saúde e resultando em uma autoavaliação ruim/péssima da saúde. ${ }^{24} \mathrm{~A}$ 
presença de diversas doenças, as possíveis interações medicamentosas ou efeitos adversos podem reforçar o autorrelato mencionado e contribuir para a má adesão ao tratamento. Sendo assim, destaca-se a importância da atuação dos profissionais de saúde também no tratamento não farmacológico e em atividades de educação em saúde, o que, consequentemente, pode contribuir com a redução da polifarmácia e melhora da percepção de saúde. ${ }^{24}$

Ainda que o tratamento farmacológico seja significativo para o controle das DCNT, o tratamento não farmacológico, como mudanças no estilo de vida, dando ênfase para a dietoterapia, é imprescindível no tratamento de adultos e idosos com tais doenças. ${ }^{24} \mathrm{O}$ alto índice de prevalência de DCNT em idosos pode demandar tratamento nutricional com prescrição de dieta e de alguns alimentos específicos, tornando-se necessário estudar a associação deles com polifarmácia. ${ }^{24}$

O uso inadequado dos medicamentos causou cerca de 30\% das intoxicações ocorridas no Brasil em 2009 e liderou, nos últimos 10 anos, a lista de reações tóxicas notificadas. ${ }^{25}$ Observou-se que é comum entre os pacientes idosos, que esqueciam de tomar os medicamentos a utilização somente no outro dia. $\mathrm{O}$ atrasado na utilização ou pular dose das medicações são condutas que trazem riscos, podendo interferir na eficácia do tratamento medicamentoso, visto que cada medicamento tem uma meia vida de ação diferente do outro. Com isso, dependendo de quanto tempo atrasado, pode ocorrer um hiato e o paciente ficar sem o medicamento neste período, podendo levar um piora nas suas comorbidades, causando até mesmo internações hospitalares. ${ }^{26}$

Os medicamentos à base de estatinas indicados para o controle do colesterol, por exemplo, normalmente são tomados à noite e, quando esquecidos por um dia, não é uma ocorrência tão grave quanto medicamentos hipoglicemiantes. Comumente, o recomendado é de que as estatinas sejam tomadas somente na noite seguinte, já a insulina, deve ser administrada assim que a pessoa se lembrar, porém,a dose deverá ser ajustada para não provocar o excesso ou diminuição da quantidade de glicose no sangue do paciente. ${ }^{26}$

Dados da Organização Mundial da Saúde (OMS) ${ }^{27}$ revelam que o percentual de internações hospitalares provocadas por reações adversas a medicamentos ultrapassa $10 \%$. As hospitalizações por uso inadequado de medicamentos no Brasil, em 2013, foram 48 milhões de atendimentos de urgência. Os problemas ligados a medicamentos provocam cada ano em Brasil, de 9-24\% das internações de urgência. ${ }^{28}$

$\mathrm{O}$ tratamento medicamentoso mostra-se um dos elementos dos quais merece um maior cuidado na atenção à saúde do idoso. O conhecimento do perfil de utilização de medicamentos é de extrema importância para traçar estratégias de uso racional de fármacos entre esse grupo etário. ${ }^{20}$ Identificar os motivos que causam o esquecimento em busca de aperfeiçoar e organizar o tratamento medicamentoso do paciente é de extrema importância para que este tenha resultados positivos no seu quadro clínico.

A adesão é um processo dinâmico e as intervenções devem ser adaptadas a cada paciente. Requer acompanhamento constante, treinamento dos profissionais de saúde e participação da família e comunidade. ${ }^{18}$

A população idosa constitui-se uma parte representativa da sociedade que se encontra mais suscetível ao consumo desregrado de medicamentos, pois, com o aumento da idade, apresentam-se declínios relativos ao envelhecimento que muitos tentam retardar através de farmacoterapia sem a recomendação de um especialista. ${ }^{29}$ Neste contexto, o uso de plantas medicinais pode ser considerado como uma forma de complementação ao tratamento medicamentoso em que não haveria necessidade de prescrição. Porém há que se considerar que o uso de tais plantas também pode acarretar riscos à saúde humana se usado utilizado de maneira inadequada.

A automedicação é uma prática bastante difundida. Em diversos países diante de a venda e propaganda de fármacos de aquisição sem prescrição médica, não há orientação adequada para aqueles que fazem uso da automedicação, sendo considerado um fator preocupante no país. ${ }^{30}$

Diante disto, observa-se a necessidade de ater-se mais à forma como as informações são veiculadas e como produtos da área farmacêutica são distribuídos, pois, apesar de os idosos serem uma porção da sociedade que mais apresentam queixas quanto à saúde, estes são os que recorrem com maior facilidade a medicamentos em busca de alívio imediato para seus problemas. ${ }^{29}$

O uso de plantas medicinais com o intuito de tratar doenças humanas é um costume histórico e muito do que se sabe é oriundo do conhecimento popular. A troca de saberes, principalmente em conversas, permitiu que várias gerações pudessem acessar a diferentes formas de tratamento baseados em plantas. ${ }^{31,32}$ Contudo, levando em conta o custo elevado destes fármacos, o difícil acesso, os seus efeitos colaterais, assim como, atualmente, o aumento do consumo de produtos de origem natural, auxiliaram no retorno do uso de plantas medicinais. ${ }^{33}$

Muitas plantas medicinais possuem compostos bioativos que apresentam boa ação farmacológica e, 
ao mesmo tempo, estão isentas dos efeitos adversos indesejáveis desencadeados pelos medicamentos utilizados repetitivamente. ${ }^{34}$ Contudo, podem surgir os efeitos indesejáveis e a maioria deles se diz respeito dos problemas no seu processamento, como a errônea identificação da planta, deficiente prática de processamento, contaminação, substituição e adulteração de planta, preparo e/ou dosagem incorretos e a falta de padronização. ${ }^{35}$

A automedicação relacionada aos fitoterápicos e plantas medicinais pode se mostrar prejudicial, visto que pode desencadeardiversas complicações como: intoxicações, aumento de resistência microbiana, mascaramento de doenças ou até mesmo a morte. Pelas plantas medicinais demonstrarem eficácia em muitos casos, isso leva a população a acreditar que sempre serão eficazes, a aparente ideia de que tais produtos são isentos de efeitos tóxicos e/ ou colaterais por serem "naturais". Contudo, várias dessas práticas podem causar danos à saúde. Estes episódios são associados à falta de informação da população nesse campo da fitoterapia. ${ }^{36,37}$ Sendo assim, o acompanhamento e orientação adequada são essenciais para segurança do paciente e de seu tratamento.

Os resultados desta pesquisa evidenciaram o frequente uso das plantas medicinais pelos participantes. Esta prática deve ser tida como um espaço de interação de saberes e práticas que valoriza: cultura, práticas e saberes locais, a interação dos usuários com a natureza e com os profissionais da equipe de saúde. Além disso pode ser uma forma de qualificar a escuta a outros saberes relacionados a comunidade, fundamentais para a promoção de saúde. Os saberes leigos, populares e tradicionais podem possibilitar a aproximação do profissional da saúde com o paciente. ${ }^{38}$

Como limitações deste estudo destaca-se o fato da coleta de dados ter sido realizada com um grupo pequeno de informantes, por se tratar de uma abordagem qualitativa, o que impossibilita generalizar os resultados para outros contextos de atendimento ao idoso em uso de polimedicação. Em vista disso, aponta-se a necessidade de ampliar a população em estudos quantitativos e em cenários diversificados de atendimento.

\section{CONSIDERAÇÕES FINAIS}

Com o aumento da expectativa de vida, e consequentemente o aumento das doenças crônicas, os idosos possivelmente são o grupo etário que mais recebe medicamentos, levando a um tratamento polimedicamentoso. Tratamento esse que, muitas vezes, é dado sem uma explicação adequada levando a uma má adesão, interação medicamentosa, reações adversas a medicamentos (RAM) e a automedicação. Com isso, esforços para aprimorar a seleção, prescrição, a dispensação e a utilização de medicamentos devem constituir prioridade nos programas de atenção ao idoso.

Pode-se observar que mais da metade dos pacientes entrevistados não tinham o ensino médio completo, o que pode estar relacionado com o baixo entendimento do tratamento, ainda mais naqueles que moram sozinhos. Condutas como identificar os medicamentos apenas pelo formato e/ou cor, não tomar no horário e dose prescritos e automedicação apresentam um grande risco de erro medicamentoso. Muitas vezes, tais erros também podem ocorrer por limitações físicas como diminuição da acuidade visual e auditiva. Com isso, a ajuda de um familiar e da equipe multidisciplinar torna-se essencial para que o mesmo alcance o objetivo do tratamento.

É necessário alterar a postura dos profissionais de saúde frente a terapia medicamentosa em idosos, para que tenha uma assistência livre de danos a esta população vulnerável. Entre algumas estratégias para reduzir a polifarmácia e a cascata de iatrogênica em idosos, pode-se elencar: conseguir um o histórico medicamentoso completo do paciente, atentando para automedicação e associações medicamentosas, prescrever apenas com indicação específica e cientificamente fundamentada e definindo claramente os objetivos da terapia proposta, além de simplificar o regime medicamentoso, quando possível. A comunicação e trabalho conjunto entre prescritores e dispensadores também permite o alcance de resultados eficientes na prevenção de RAM, beneficiando significativamente a saúde do paciente idoso.

A respeito do uso de plantas medicinais concomitante ao tratamento medicamentoso, pode-se notar que ainda existem poucos estudos que abordam as possíveis interações destas com os fármacos. Porém, é importante orientar que ervas com mesmo efeito das medicações deve ser evitadas, para evitar potencialização do efeito do fármaco e podendo causar efeitos adversos ou até mesmo hospitalizações. Deste modo, nota-se a necessidade de mais pesquisas interdisciplinares sobre o assunto.

Entender a rotina, os hábitos, crenças e conhecimentos que o paciente possui frente as suas comorbidades e tratamento são fatores que devem ser analisados no momento de auxiliar este idoso a organizar seu tratamento medicamentoso a fim de facilitar a adesão. Sendo assim, este estudo poderá contribuir para o atendimento ambulatorial a essa população, visando melhoria nas estratégias para 
explicar e ajudar este paciente a seguir o tratamento de forma correta. Diante das consultas de enfermagem, o enfermeiro pode auxiliar estes pacientes a organizar seus medicamentos quanto às dosagens, armazenamento e horários corretos, além de orientar sobre o uso de ervas medicinais concomitantes ao tratamento, com o intuito de ajudá-los a melhor compreender seu tratamento medicamentoso.

\section{REFERÊNCIAS}

1. Rocha BS, Werlang MC. Psicofármacos na estratégia saúde da família: perfil de utilização, acesso e estratégias para a promoção do uso racional. Ciênc Saúde Coletiva. 2013;18(11):3291-300. https://doi.org/10.1590/s141381232013001100019

2. Silva JVF, Silva EC, Rodrigues APRA, Miyazawa AP. A relação entre o envelhecimento populacional e as doenças crônicas não transmissíveis: sério desafio de saúde pública. Ciências Biológicas e da Saúde. 2015;2(3):91-100. https:// doi.org/10.1590/1413-81232018235.31552017

3. World Health Organization. Noncommunicable Diseases Progress Monitor 2017 [Internet]. Geneva: WHO; 2017 [capturado em 24 jul. 2019]. Disponível em: https:// www.who.int/nmh/publications/ncd-progress-monitor2017/en/.

4. Brasil. Ministério da Saúde. Secretaria de Ciência, Tecnologia e Insumos Estratégicos. Departamento de Ciência e Tecnologia. Síntese de evidências para políticas de saúde: adesão ao tratamento medicamentoso por pacientes portadores de doenças crônicas. Brasília: Ministério da Saúde; 2016. https://doi.org/10.1590/0103. 1104201711217

5. Malta DC, Silva Jr JB. O Plano de ações estratégicas para o enfrentamento das doenças crônicas não transmissíveis no brasil e a definição das metas globais para o enfrentamento dessas doenças até 2025: uma revisão. Epidemiol Serv Saúde. 2013;22(1):151-64. https://doi. org/10.5123/s1679-49742013000100016

6. Magnabosco P, Teraoka EC, Oliveira EM, Felipe EA, Freitas D, Marchi-Alves LM. Análise comparativa da não adesão ao tratamento medicamentoso da hipertensão arterial sistêmica em população urbana e rural. Rev Latino-Am Enfermagem. 2015;23(1):20-7. https://doi. org/10.11606/t.22.2015.tde-29052015-193755

7. Santos MVR, Oliveira DC, Arraes LB, Medeiros L, Novaes MA. Adesão ao tratamento anti-hipertensivo: conceitos, aferição e estratégias inovadoras de abordagem. Rev Bras Clín Med. 2013;11(1):55-61.

8. Brasil. Ministério da Saúde. Secretaria de Ciência, Tecnologia e Insumos Estratégicos. Departamento de Ciência e Tecnologia. Síntese de evidências para políticas de saúde: adesão ao tratamento medicamentoso por pacientes portadores de doenças crônicas. Brasília: Ministério da Saúde; 2016 https://doi.org/10.1590/0103. 1104201711217

9. Rosa GR, Camargo EAF. Polimedicação em idosos. Interciência \& Sociedade. 2014;3(2):72-8.
10. Remondi FA, Cabrera MAS, Souza RKT. Não adesão ao tratamento medicamentoso contínuo: prevalência e determinantes em adultos de 40 anos e mais. Cad Saúde Pública. 2014;30(1):126-36. https://doi.org/10.1590/0102$311 \times 00092613$

11. Girotto E, Andrade SM, Cabrera MAS, Matsuo T. Adesão ao tratamento farmacológico e não farmacológico e fatores associados na atenção primária da hipertensão arterial. Ciênc Saúde Coletiva. 2013;18(6):1763-72. https://doi. org/10.1590/s1413-81232013000600027

12. Oliveira FP. Fatores que interferem na adesão ao tratamento anti-hipertensivo pelos idosos: revisão de literatura [monografia] [Internet]. Araçuaí: UFMG; 2013 [capturado em 24 jul. 2019]. Disponível em: https://www. nescon.medicina.ufmg.br/biblioteca/imagem/4108.pdf. https://doi.org/10.11606/d.5.2014.tde-24062014-141705

13. Minayo MCS. Pesquisa social: teoria, método e criatividade. Rio de Janeiro: Vozes; 2010. v. 29.

14. Polit DF, Beck CT. Fundamentos de pesquisa em enfermagem: avaliação de evidência para a prática da enfermagem. 7. ed. Porto Alegre: Artmed; 2011.

15. Carvalho MFC, Romano-Lieber NS, Bergsten-Mendes G, Secoli SR, Ribeiro E, Lebrão ML, Duarte YAO. Polifarmácia entre idosos do Município de São Paulo Estudo SABE. Rev Bras Epidemiol. 2012;15(4):817-27. https://doi.org/10.1590/s1415-790x2012000400013

16. Silva MRR, Diniz LM, Santos JBR, Reis EA, Mata AR, Araújo VE, Álvares J, Acurcio FA. Uso de medicamentos e fatores associados à polifarmácia em indivíduos com diabetes mellitus em Minas Gerais, Brasil. Ciênc Saúde Coletiva. 2018;23(8):2565-74. https://doi. org/10.1590/1413-81232018238.10222016

17. Domingues GR, Alves KO, Carmo PHS, Galvão SS, Teixeira SS, Baldoino EF. A atuação do psicólogo no tratamento de pacientes terminais e seus familiares. Psicol Hosp. 2013;11(1):2-24.

18. UNA-SUS/UERJ. Uso de medicamentos em idosos [Internet]. 2017 [capturado em 2 jun. 2018]. Disponível em: https://ares.unasus.gov.br/acervo/handle/ARES/7793.

19. Neves SJF, Marques AP, Leal MCC, Diniz AD, Medeiros TS, Arruda IKG. Epidemiologia do uso de medicamentos entre idosos em área urbana do Nordeste do Brasil. Rev Saúde Pública. 2013;47(4):759-68. https://doi.org/10.1590/ s0034-8910.2013047003768

20. Lobo LB. Polifarmácia entre os idosos de dourados, Mato Grosso do Sul: um estudo de base populacional [dissertação] [Internet]. Dourados: UFGD; 2015 [capturado em 15 ab. 2018]. Disponível em: http:// files.ufgd.edu.br/arquivos/arquivos/78/MESTRADO. DOUTORADO-CIENCIAS-SAUDE/27.06.15\%20 TESE\%20LIGIA\%20(PT\%202)\%20defesa.pdf. https://doi.org/10.21475/ajcs.2016.10.08.p7408

21. Hall JE. Guyton \& Hall: tratado de fisiologia médica. 13. ed. Rio de Janeiro: Elsevier; 2017.

22. Torriani MS, Santos L, Echer IC, Barros E, organizadores. Medicamentos de A a Z 2016-2018: enfermagem. Porto Alegre: Artmed; 2016. 
23. Oriá RB, Brito GAC. Sistema digestório: integração básico-clínica [Internet]. São Paulo: Blucher; 2016 [capturado em 30 maio 2018]. Seção 5, cap. 31. Disponível em: https://openaccess.blucher.com.br/articlelist/sistema-digestorio-317/list\# committee. https://doi.org/10.5151/9788580391893

24. Silveira EA, Dalastra L, Pagotto V. Polypharmacy, chronic diseases and nutritional markers in community-dwelling older. Rev Bras Epidemiol. 2014;17(4):818-29. https://doi. org/10.1590/1809-4503201400040002

25. Fiocruz. Sistema Nacional de Informações TóxicoFarmacológicas [Internet]. Rio de Janeiro: Fiocruz; c2009 [capturado em 2 jun. 2018]. Disponível em: http:// www.fiocruz.br/sinitox. https://doi.org/10.17143/ciaed/ xxiilciaed.2017.00386

26. Corradi MFDB. Vai começar um tratamento? Pergunte ao médico o que fazer se esquecer-se de tomar o medicamento na hora certa [Internet]. São Paulo: Hospital Sírio-Libanês; 2015 [capturado em 5 jun. 2018]. Disponível em: https:/www.hospitalsiriolibanes.org.br/suasaude/Paginas/vai-comecar-tratamento-pergunte-medicofazer-esquecer-tomar-medicamento-hora-certa.aspx. https://doi.org/10.1590/s0102-88392001000300002https:// doi.org/10.1590/s0102-88392001000300002

27. World Health Organization. Envelhecimento ativo: uma política de saúde. Brasília: Organização Pan-Americana da Saúde; 2005.

28. Brasil. Ministério da Saúde. Fundo Nacional de Saúde Fundo [Internet]. Brasília: Ministério da Saúde; 2014 [capturado em 24 jul. 2019]. Disponível em: http:// portalfns.saude.gov.br/. https://doi.org/10.1590/s0034. 89102006000500029

29. Luz DJ, Lima JAS, Monteiro LG. Automedicação no idoso [monografia] [Internet]. Mindelo: Universidade do Mindelo; 2013 [capturado de 31 maio 2019]. Disponível em: http://www. portaldoconhecimento.gov.cv/bitstream/10961/3252/1/ Luz\%2C\%20Lima\%20e\%20Monteiro\%202013.\%20 Automedica\%C3\%A7\%C3\%A30\%20no\%20Idoso.pdf.

30. Both JS, Kauffmann C, Ely LS, Dall'agnol R, Rigo MPM, Teixeira MFN, Castro LC. Cuidado farmacêutico domiciliar ao idoso: análise de perfil e necessidades de promoção e educação em saúde. Cad Pedagógico. 2015;12(3):66-84.

31. Siqueira JBV, Teila Ceolin, Ceolin S, Minuto JC, Oliveira SG, Oliveira ADL. Uso de plantas medicinais por hipertensos e diabéticos de uma estratégia saúde da família rural. Rev Contexto Saúde. 2017;17(32):33-45. https://doi. org/10.21527/2176-7114.2017.32.33-45

32. Silva FA. Tratamento do diabetes mellitus tipo 2 através do uso de plantas medicinais [monografia] [Internet]. Ariquemes: FAEMA; 2017 [capturado em 5 maio 2018]. Disponível em: http://repositorio.faema.edu.br:8000/ handle/123456789/1253. https://doi.org/10.31072/rcf. v9iedesp.616

33. Balbinot S, Velasquez PG, Düsman E. Reconhecimento e uso de plantas medicinais pelos idosos do Município de Marmeleiro - Paraná. Rev Bras Plantas Med. 2013;15 (4 Suppl 1):632-8. https://doi.org/10.1590/s151605722013000500002
34. Bahmani M, Zargaran A, Rafieian-Kopaei M, Sakid K. Ethnobotanical study of medicinal plants used in the management of diabetes mellitus in the Urmia, Northwest Iran. Asian Pac J Trop Med. 2014;7(S1):S348-54. https:// doi.org/10.1016/s1995-7645(14)60257-1

35. Vieira LG. O uso de fitoterápicos e plantas medicinais por pacientes diabéticos [monografia] [Internet]. Brasília: UnB; 2017 [capturado em 12 dez. 2017]. Disponível em: http://bdm.unb.br/bitstream/10483/17579/1/2017 LiviaGumieriVieira.pdf.

36. Brito AF. Automedicação com produtos naturais entre os acadêmicos da Facer, Unidades Ceres-GO e RubiatabaGO. Ceres: Facer; 2014.

37. Machado HL, Moura VL, Gouveia NM, Costa GA, Espindola FS, Botelho FV. Pesquisa e atividades de extensão em fitoterapia desenvolvidas pela Rede FitoCerrado: uso racional de plantas medicinais e fitoterápicos por idosos em Uberlândia-MG. Rev Bras Pl Med. 2014; 16(3):527-33. https://doi.org/10.1590/1983084x/13_072

38. Antonio GD, Tesser CD, Moretti-Pires RO. Contribuição das plantas medicinais para o cuidado e a promoção da saúde na atenção primária. Interface. 2013;17(46):615-33. https://doi.org/10.1590/s1414-32832013005000014

\section{AUTHORS:}

RENATTA RossatTo de ARAúJo

Enfermeira. Graduada pela Universidade Federal do Rio Grande do Sul (UFRGS).

E-mail: renattarossatto@hotmail.com

Orcid: https://orcid.org/0000-0003-2949-7885

ANA KaRINA SIIVA DA ROCHA TANAKa

Enfermeira. Graduada pela Universidade do Vale do Rio dos Sinos (UNISINOS). Mestrado em Gerontologia Biomédica na Pontifícia Universidade Católica do Rio Grande do Sul (PUCRS). Doutora em Gerontologia Biomédica na PUCRS, Professora da Escola de Enfermagem, Universidade Federal do Rio Grande do Sul. Editora Associada da Revista Gaúcha de Enfermagem.

E-mail: ana.tanaka@ufrgs.br; anakarinatanaka@gmail.com

Orcid: https://orcid.org/0000-0003-2488-3656

LUIZA MARIA GERHARDT

Enfermeira. Graduada pela Universidade Federal do Rio Grande do Sul (1976). Doutorado em Educação pela Pontifícia Universidade Católica do Rio Grande do Sul (2005). Professora da Universidade Federal do Rio Grande do Sul.

E-mail: luizam1928@gmail.com

Orcid: https://orcid.org/0000-0003-1558-381 1

MaRIA LUIZA PAZ MACHADO

Enfermeira. Graduada pela Universidade de Enfermagem Nossa Senhora Medianeira (1985). Mestrado em Enfermagem pela Universidade Federal do Rio Grande do Sul (2000) e doutorado em Enfermagem pela Universidade Federal do Rio Grande do Sul (2013). Atualmente Professora da Universidade Federal do Rio Grande do Sul.

E-mail: luiza@enf.ufrgs.br

Orcid: https://orcid.org/0000-0002-2213-7871 